\title{
SÍNTESE DE NANOTUBOS DE CARBONO PELO MÉTODO CVD ANALISANDO OS PARÂMETROS DE SÍNTESE VIA PLANEJAMENTO FATORIAL DE EXPERIMENTOS
}

\author{
T. LEONARDI ${ }^{1}$, M. MARCHESIN ${ }^{1}$, J. R. BARTOLI ${ }^{1}$, I. PEREYRA ${ }^{2}$, M. N .P. CARREÑO ${ }^{2}$, I. ABE $^{2}$ \\ ${ }^{1}$ Universidade Estadual de Campinas, Departamento de Engenharia de Materiais e de Bioprocessos - \\ Faculdade de engenharia Química \\ ${ }^{2}$ Universidade de São Paulo, Laboratório de Microeletrônica - Departamento de Engenharia de \\ Sistemas Eletrônicos Escola Politécnica \\ E-mail para contato: tiagoleonardi@hotmail.com
}

\begin{abstract}
RESUMO - Os Nanotubos de Carbono (NTCs) têm sido reconhecidos, desde seu descobrimento por lijima em 1991, como materiais de grande interesse em nanotecnologia, devido às suas excelentes propriedades mecânicas, elétricas, térmicas, entre outras. Desde sua descoberta, os parâmetros de síntese têm sido estudados em busca de rotas que elevem a produtividade e a qualidade dos mesmos. Alguns trabalhos recentes reportam a melhoria na produtividade e na qualidade dos NTCs quando catalisadores contendo dois metais de transição são utilizados na síntese com reator tipo CVD (Chemical Vapour Deposition). Ainda que interessantes resultados tenham sido obtidos, estes trabalhos carecem de experimentos estatisticamente planejados para avaliar a existência ou não de interações significativas entre os parâmetros de síntese dos NTCs. Portanto, o objetivo desta pesquisa é explorar as rotas de síntese via CVD utilizando dois tipos de catalisadores binários, Ni/Co e Co/Mn, utilizando o planejamento fatorial de experimentos $2^{3}$ (FED - Factorial Experimental Design), avaliando três parâmetros de processo: temperatura, razão de fluxo de $\mathrm{CH}_{4} / \mathrm{N}_{2}$ e tempo de síntese. As repostas obtidas, como: diâmetro dos NTCs, quiralidade e pureza, serão tratados utilizando-se o software Statistica $^{\mathrm{TM}}$, verificando as interações entre estes parâmetros e suas respectivas significâncias, buscando otimizar a síntese de nanotubos.
\end{abstract}

\section{INTRODUÇÃO}

Nanotubos de Carbono (NTCs) foram descritos, pela primeira vez, por Iijima (1991) e segundo Belin e Epron (2005), desde sua descoberta, eles têm contribuído para o desenvolvimento de estudos nas áreas da física, química e ciência dos materiais e têm sido um material extremamente promissor. Kumar e Ando (2010) citam que suas propriedades extraordinárias abriram uma ampla gama de aplicações e desencadearam uma corrida ao ouro na academia e laboratórios industriais por todo o mundo na busca por usos práticos dos NTCs. Odom et al. comentaram sobre as distintas características do NTCs que aparecem devidas á sua estrutura atômica e suas dimensões nanométricas. Por exemplo, eles podem ser metálicos ou 


\section{9 a 22 de outubro de 2014 \\ Florianópolis/SC}

semicondutores, dependendo da quiralidade e do diâmetro. Cheung et al. mostraram que o diâmetro dos NTCs é diretamente proporcional ao tamanho das partículas dos catalisadores, influenciando também nas propriedades mecânicas e elétricas dos NTCs e, por isso, o interesse no controle da síntese é de suma importância.

Podem ser subdivididos em dois grupos, os de paredes múltiplas, Nanotubos de carbono paredes múltiplas (NTCPMs) e os Nanotubos de carbono parede simples (NTCPSs), conforme mostra a Figura 1.
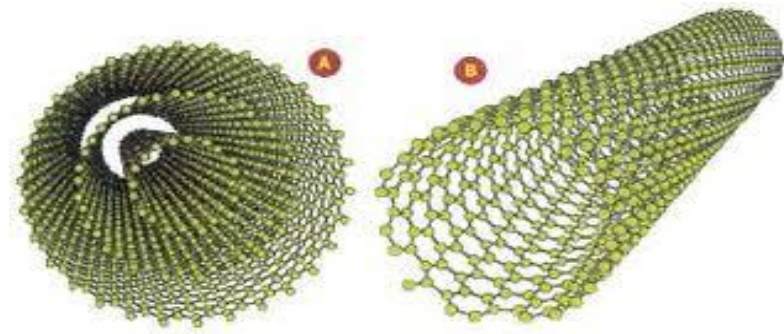

Figura 1 - Nanotubos de carbono (A) multicamadas, (B) camada simples.

Os NTCPSs são de maior importância, devido á sua maior estabilidade estrutural, e possuem melhores propriedades e perpectivas de aplicação, porém são mais difíceis de serem obtidos do que os NTCPMs. Já os NTCPMs são formados por duas ou mais camadas atômicas de carbono enroladas de forma concêntricas, com espaçamento entre os tubos próximos à distância intercamada da grafita $(0,34 \mathrm{~nm})$ e são mantidos juntos através de interações do tipo Van der Waals.

Dentre os métodos de síntese (Descarga-arco, Ablação a laser e CVD), o CVD (Chemical vapor deposition) é o mais utilizado atualmente, pois necessita de temperaturas não tão elevadas e confere maior controle durante a síntese a um menor custo. $\mathrm{O}$ processo CVD consiste em vários passos. O primeiro é preparar as nanoparticulas de metal no substrato. O substrato é então colocado no forno $\left(500^{\circ} \mathrm{C}\right.$ a $\left.1200^{\circ} \mathrm{C}\right)$ e as nanopartículas são, geralmente, submetidas a um tratamento de redução. Finalmente, um precursor de carbono é introduzido no forno e a deposição de carbono ocorre pela decomposição catalítica das moléculas do precursor de carbono sobre as nanoparticulas de metal explica Dupuis (2005). Hidrogênio é liberado na forma de gás e o carbono se dissolve no catalisador. Quando o limite de solubilidade é atingido, o excesso de carbono presente no catalisador é precipitado na forma de uma estrutura cilíndrica. Os metais mais comumente usados são $\mathrm{Fe}, \mathrm{Co}, \mathrm{Ni}$, devido a duas razões: (i) Alta solubilidade do carbono nestes metais em altas temperaturas; e (ii) Alta taxa de difusão do carbono nestes metais de acordo com Kumar (2011).

Recentemente, Rodrigues et al.(2011) utilizaram etileno como precursor de carbono para crescimento de NTCs via CVD, os quais foram funcionalizados e caracterizados via XPS. Abe $e t$ al. estudaram o crescimento de nanotubos de carbono sobre substrato de $\mathrm{Si}$ e $\mathrm{SiO}_{2}$, utilizando $\mathrm{Ni}$, como catalisador, depositado ou via Sputtering ou via electron beam. Eles utilizaram a fotolitografia (processo lift-off) nos filmes de Ni para delimitar padrões geométricos e avaliar a eficiência dos catalisadores. É crescente o número de trabalhos avaliando o crescimento de 


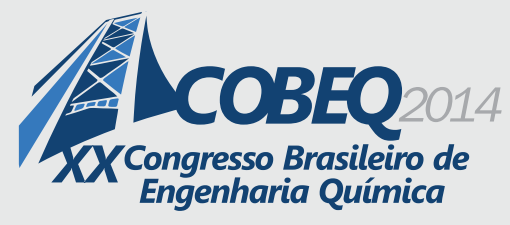

19 a 22 de outubro de 2014
Florianópolis/SC

nanotubos sobre catalisadores contendo dois metais e os resultados mostram maior produtividade e menor temperatura necessária para síntese. Em experimento conduzido por Ferlauto et al. o catalisador yttria-estabilizada/zircônia/níquel (YSZ/Ni) foi utilizado para obtenção de NTCPMs de alta qualidade. Li et al. (2005) e Zhan et al. (2007) reportaram um novo catalisador dual composto por $\mathrm{Ni}-\mathrm{Mo} / \mathrm{MgO}$, para produção de NTCPMs com alta eficiência e pureza em torno de $95 \%$.

Atualmente, além de rotas de síntese, muito se tem estudado sobre as aplicações dos nanotubos de carbono. Marchesin e Cecci (2012) têm estudado o desenvolvimento de nanocompósitos de PMMA/NTCPM para aplicações em dispositivos optoeletrônicos e células solares fotovoltaicas. Ladeira et al (2009) depositaram pedido de patente sobre o processo de síntese continua, em larga escala, de nanotubos de carbono com clínquer de cimento como substrato, buscando uma forma de cimento nanoestruturado com NTCs.

Embora bons resultados tenham sido obtidos nestes trabalhos, em termos de crescimento de NTCs observa-se uma carência de procedimentos experimentais planejados estatisticamente, isto é, as variáveis de processo não são avaliadas simultaneamente. Isto impede conhecer possíveis interações significativas entre as variáveis e obter um modelo matemático válido dentro das condições de contorno em estudo. Portanto, esta sendo proposto um planejamento fatorial $2^{3}$, com ponto central a três repetições (estimativa do erro) para a síntese de NTCs via processamento em forno CVD, utilizado dois tipos de catalisadores binários, $\mathrm{Ni} / \mathrm{Co}$ e $\mathrm{Co} / \mathrm{Mn}$. O objetivo é verificar a hipótese de qual rota com tais catalisadores é eficaz e otimizada na síntese de nanotubos de carbono. Utilizando-se o software Statística [20], as respostas do planejamento experimental: rendimento de carbono, taxa de crescimento média e a conversão de metano, serão analisados, podendo-se obter um modelo matemático válido nas condições de contorno estabelecidas pelos níveis das variáveis escolhidas, bem como as superfícies de resposta e curvas de contorno das variáveis.

\section{Materiais e Métodos}

Todas as amostras foram preparadas num reator tubular CVD de aquecimento por resistência revestido com cerâmica refrataria, da marca Kokusai - Tokyo Japan sendo o leito, um tubo de quartzo de duas polegadas de diametro e $70 \mathrm{~cm}$ de comprimento, situado no laboratório de microeletrônica da faculdade de eng. elétrica POLI-USP. Como suporte para os catalisadores serão utilizadas placas de quartzo de uma polegada quadrada de área e $1 \mathrm{~mm}$ de espessura (Dykrom). Para síntese dos catalisadores os seguintes sais serão utilizados: Acetato de Ni tetrahidratado $\left(\mathrm{C}_{4} \mathrm{H}_{6} \mathrm{NiO}_{4} .4 \mathrm{H}_{2} \mathrm{O}\right)$ (P.A.), Acetato de Co II $\left(\mathrm{C}_{4} \mathrm{H}_{6} \mathrm{CoO}_{4} \cdot 4 \mathrm{H}_{2} \mathrm{O}\right)$ (P.A.) e Acetato de Mn II ( $\left.\left(\mathrm{CH}_{3} \mathrm{CO}_{2}\right)_{2} \mathrm{Mn} .4 \mathrm{H}_{2} \mathrm{O}\right)$ (P.A.) (Vetec). Os gases utilizados durante a síntese serão o $\mathrm{N}_{2}$ $(99,995 \%)$ e o $\mathrm{CH}_{4}(99,999 \%)$.

Na preparação dos catalisadores, Acetato de Co II e Acetato de Mn II ou Acetato de Ni $(1: 1 \mathrm{M})$ e etileno glicol ficam sob agitação constante e refluxo a $90{ }^{\circ} \mathrm{C}$ por $5 \mathrm{~h}$. A solução é seca a $200{ }^{\circ} \mathrm{C}$ por $24 \mathrm{~h}$, obtendo-se um pó roxo. Então, este pó é macerado em gral e peneirado em uma peneira de 325 mesh. O catalisador é disperso no substrato e seu peso é registrado. Substrato + 
catalisador são colocados no forno e aquecidos a $700^{\circ} \mathrm{C}$, sob atmosfera de $\mathrm{N}_{2}$, por $1 \mathrm{~h}$. Após a calcinação, a amostra é aquecida até a temperatura desejada (conforme planejamento fatorial mostrado na Tabela 1.) e o fluxo gás $\mathrm{CH}_{4}$ é, então, liberado na razão desejada $\mathrm{CH}_{4} / \mathrm{N}_{2}$. Espera-se o tempo de síntese pré-definido no planejamento. A amostra é então resfriada até $250^{\circ} \mathrm{C}$, sob atmosfera de $\mathrm{N}_{2}$, e posteriormente é retirada do forno e pesada à temperatura ambiente.

As variáveis e os níveis estudados são mostrados na Tabela 1 e o planejamento fatorial $2^{3}$, totalizando 8 ensaios +3 pontos centrais) é mostrado na Tabela 2, estes parâmetros de síntese de NTC via CVD são usados para as duas rotas com os catalisadores binários Ni/Co e Co/Mn.

Tabela 1 - Níveis e fatores a serem estudados no planejamento experimental.

\begin{tabular}{lcccc}
\hline & Variáveis & \multicolumn{3}{c}{ Níveis } \\
\hline & & $\mathbf{- 1}$ & $\mathbf{0}$ & $\mathbf{1}$ \\
$\mathbf{X}_{\mathbf{1}}$ & $\mathbf{T}\left({ }^{\circ} \mathbf{C}\right)$ & 850 & 900 & 950 \\
$\mathbf{X}_{\mathbf{2}}$ & $\mathbf{C H} / \mathbf{N}_{\mathbf{2}}$ & $1: 1$ & $2: 1$ & $3: 1$ \\
$\mathbf{X}_{\mathbf{3}}$ & $\mathbf{T e m p o}(\mathbf{m i n})$ & 5 & 15 & 25 \\
\hline
\end{tabular}

Tabela 2 - Planejamento experimental $2^{3}$ com ponto central a três repetições.

\begin{tabular}{ccccccc}
\hline & \multicolumn{3}{c}{ Níveis Codificados } & \multicolumn{3}{c}{ Respectivos Valores } \\
\hline Ensaio & $\mathbf{X}_{\mathbf{1}}$ & $\mathbf{X}_{\mathbf{2}}$ & $\mathbf{X}_{\mathbf{3}}$ & $\begin{array}{c}\text { Temperatura } \\
\left({ }^{\circ} \mathbf{C}\right)\end{array}$ & $\begin{array}{c}\mathbf{C H}_{\mathbf{4}} / \mathbf{N}_{\mathbf{2}} \\
\text { proporção }\end{array}$ & $\begin{array}{c}\text { Tempo } \\
(\mathbf{m i n})\end{array}$ \\
1 & -1 & -1 & -1 & 850 & $1: 1$ & 5 \\
2 & 1 & -1 & -1 & 950 & $1: 1$ & 5 \\
3 & -1 & 1 & -1 & 850 & $3: 1$ & 5 \\
4 & 1 & 1 & -1 & 950 & $3: 1$ & 5 \\
5 & -1 & -1 & 1 & 850 & $1: 1$ & 25 \\
6 & 1 & -1 & 1 & 950 & $1: 1$ & 25 \\
7 & -1 & 1 & 1 & 850 & $3: 1$ & 25 \\
8 & 1 & 1 & 1 & 950 & $3: 1$ & 25 \\
9 & 0 & 0 & 0 & 900 & $2: 1$ & 15 \\
10 & 0 & 0 & 0 & 900 & $2: 1$ & 15 \\
11 & 0 & 0 & 0 & 900 & $2: 1$ & 15 \\
\hline
\end{tabular}

Testes para determinação da área superficial específica e porosidade por adsorção física de $\mathrm{N}_{2}$ (BET/ASAP), MEV_EDS e DRX serão realizados, podendo-se assim, prever a qualidade dos catalisadores. Os nanotubos estão sendo caracterizados via Microscopia eletrônica de varredura (MEV), Microscopia eletrônica de transmissão (MET), Difração de raio-x (DRX), espectroscopia Raman, Análise termogravimétrica (TGA). O equipamento Raman usado é um WITEC, Confocal Raman Microscope Alpha300 R, para as lentes com aumento de x10 e x50, com laser de $532 \mathrm{~nm}$. As análises MET foram feitas no microscópio JEOL JEM 2100F HRP, com tensão de aceleração de 300 kV e faixa de ampliação de 50 a 800 mil vezes com fonte de elétrons de LaBF6. 
$\mathrm{O}$ rendimento de carbono, taxa de crescimento média e a conversão de metano serão calculados usando as seguintes Equações (1), (2) e (3), respectivamente.

$$
\begin{aligned}
& =\frac{m_{\text {total }}-m_{\text {cat }}}{m_{\text {cat }}} \times 100 \% \\
& =\frac{m_{\text {Total }}-m_{\text {cat }}}{\text { tempo de crescimento } \quad(\mathrm{mg} / \mathrm{min})} \\
& =\frac{m_{\text {total }}-m_{\text {cat }}}{\text { tx escoamento }(\mathrm{L} / \mathrm{min}) \times t(\mathrm{~min}) \div 22.4(\mathrm{~L} / \mathrm{mol}) \times 12(\mathrm{~g} / \mathrm{mol}) \times 100 \%}
\end{aligned}
$$

Onde: $\mathrm{m}_{\text {cat }}=$ Massa inicial do catalisador

$\mathrm{m}_{\text {total }}=$ Massa do produto + catalisador

\section{Resultados e Discussões}

Os experimentos do planejamento fatorial proposto na Tabela 1 estão em desenvolvimento para a rota utilizando o catalisador $\mathrm{Co} / \mathrm{Mn}$.

As amostras estão sendo caracterizadas pelas técnicas de espectroscopia Raman e microscopia eletrônica de transmissão (MET). Através destas técnicas podem-se avaliar características importantes dos NTCs, como: Diâmetro, número de paredes, quiralidade, pureza.

A espectroscopia Raman é a mais valiosa e tem proporcionado informações importantes sobre a estrutura dos nanotubos. Confirmou-se a formação de NTC em todas as amostras. A Figura 2 mostra o espectro referente ao ensaio 10, os espectros das demais amostras apresentam bandas de absorção nas mesmas frequências mas com variações nas intensidades. A banda larga em torno de $1340 \mathrm{~cm}^{-1}$, é atribuida às estruturas grafíticas desordenadas, chamada banda $\mathrm{D}(\mathrm{D}$ desordem), a banda de alta frequencia entre 1500 e $1600 \mathrm{~cm}^{-1}$, chamado de banda G, que é associada à nanotubos perfeitos e um pico harmônico da banda $\mathrm{D}$, chamado $\mathrm{D}^{\prime}$ entre 2450 e 2650 $\mathrm{cm}^{-1}$. Através desta técnica pode-se avaliar o grau de grafitização ou pureza do material, utilizando-se a razão entre as intensidades das bandas $\mathrm{D}$ e $\mathrm{G}$ que reflete a proporção de nanotubos perfeitos numa amostra. Quanto menor for essa razão, maior o grau de grafitização do material e, portanto, melhor a qualidade dos nanotubos formados. 


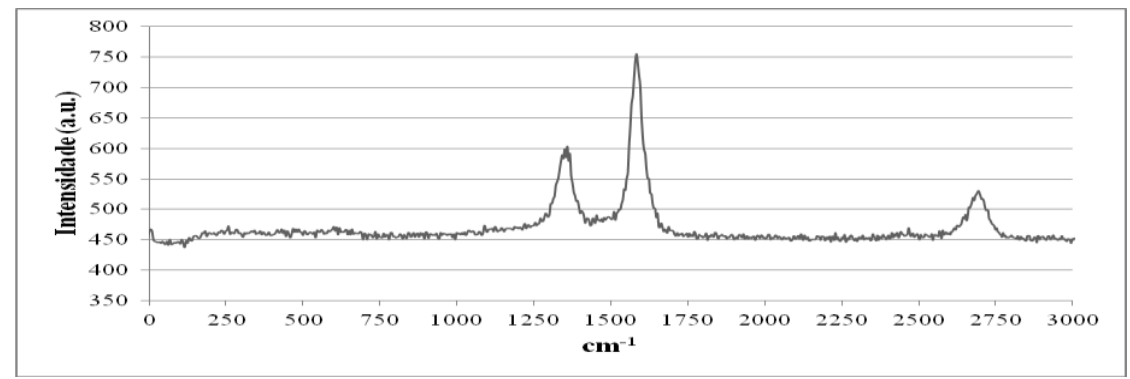

Figura 2. Espectro RAMAN da amostra sintetizada nas condições do ensaio 10.

A razão entre as intensidades $\left(\mathrm{I}_{\mathrm{D}} / \mathrm{I}_{\mathrm{G}}\right)$ para as amostras sintetizadas estão próximas a 1 , fato que revela alto grau de desordem estrutural e baixa pureza, como mostrado na Tabela 3. As amostras que mostraram um melhor resultado $\mathrm{I}_{\mathrm{D}} / \mathrm{I}_{\mathrm{G}}$, serão quimicamente purificadas e novamente caracterizadas, via Raman, para fins de comparação com as amostras não purificadas. Espera-se assim, uma diminuição do pico D e uma melhora na razão entre as intensidades devido ao aumento na pureza da amostra.

Tabela 3 - Valores de Razão $\mathrm{I}_{\mathrm{D}} / \mathrm{I}_{\mathrm{G}}$.

\begin{tabular}{|c|c|}
\hline \multirow{2}{*}{ Amostra } & Razão Intensidade \\
\cline { 2 - 2 } & $\mathrm{I}_{\mathrm{D}} / \mathrm{I}_{\mathrm{G}}$ \\
\hline 1 & 0,90 \\
\hline 2 & 0,95 \\
\hline 3 & 0,84 \\
\hline 4 & 0,89 \\
\hline 5 & 0,93 \\
\hline 6 & 0,89 \\
\hline 7 & 0,83 \\
\hline 8 & 0,86 \\
\hline 9 & 0,89 \\
\hline 10 & 0,80 \\
\hline 11 & 0,87 \\
\hline
\end{tabular}

As imagens de MET são de extrema importância, pois, possibilita a obtenção de medidas do diâmetro interno e externo de nanotubos de carbono de múltiplas camadas, número de paredes e espaçamento intercamadas. A análise via MET foi realizada na mesma amostra, obtida nas condições do ponto central do planejamento. Uma porção da amostra foi ultrassonicamente dispersa em metanol. Uma gota desta solução foi então depositada sobre uma grelha de cobre de malha 400 microscopia coberto com um filme de carbono. A imagem mostra algumas estruturas de NTC, com aproximadamente $10 \mathrm{~nm}$ de diâmetro, carbono amorfo e partículas de catalisador encapsuladas pelos nanotubos, conforme a Figura 3. 


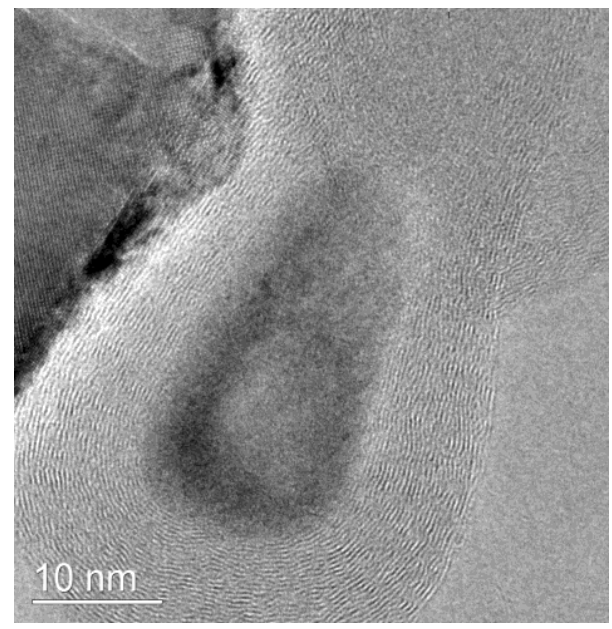

Figura 3. Imagem de MET de uma partícula de catalisador encapsulada pelo NTC sintetizada nas condições do ensaio 10.

As caracterizações das amostras da síntese de NTC obtidas nos demais experimentos do planejamento fatorial $2^{3}$ estão em desenvolvimento conforme metodologia proposta.

\section{CONCLUSÕES}

Os NTC foram obtidos, conforme análises Ramam e MET, nos primeiros resultados da rota de síntese via CVD para o sistema de catalisador binário $\mathrm{Ni} / \mathrm{Co}$, nas condições de $900{ }^{\circ} \mathrm{C}$, fluxo $\mathrm{CH}_{4} / \mathrm{N}_{2}$ em 2:1 durante 15 min (ponto central do planejamento fatorial $2^{3}$ ).

Os experimentos de síntese e caracterizações prosseguem conforme planejamento proposto para determinar o rendimento dos catalisadores em estudo $\mathrm{Ni} / \mathrm{Co}$ e $\mathrm{Co} / \mathrm{Mn}$ bem como a qualidade dos NTC produzidos.

A razão entre as intensidades $\left(\mathrm{I}_{\mathrm{D}} / \mathrm{I}_{\mathrm{G}}\right)$, no espectro Raman, revela um alto grau de desordem estrutural e baixa pureza das amostras, o que, pode ser melhorado através de métodos de purificação.

\section{AGRADECIMENTOS}

Ao CNPQ pela bolsa de mestrado.

\section{REFERÊNCIAS}

ABE, I.Y.; SILVA, D. E. L.; PREYRA, I. SBMicro, 2013.

BELIN,T.; EPRON, F. Characterization methods of carbon nanotubes: a review. Mat. Sci. and Eng., 2005, B 119, p.105-118.

CHEUNG, C. L.; KURTZ, A.; PARK, H.; LIEBER, C. M. Diameter-Controlled Synthesis of

Carbon Nanotubes. J. Phys. Chem., v.106, p. 2429-2433, 2002. 
DUPUIS, A. C. The catalyst in the CCVD of carbon nanotubes-a review. Progress in Mat. Sci., 2005, v. 50, p. 929-961.

FERLAUTO, A. S. Ferlauto; DEFLORIO, D. Z.; FONSECA, F. C.; ESPOSITO, V.; MUCCILLO, R.; TRAVERSA, E.; LADEIRA, L. O.; Chemical vapor deposition of multiwalled carbon nanotubes from nickel/yttria-stabilized zirconia catalysts. Appl. Phys. A 2006, v. 84, p. 271-276.

IIJIMA, S. Helical microtubules of graphitic carbon. Nature, v.354, p. 56-58.

KUMAR, K.; ANDO, Y. Chemical Vapor Deposition of Carbon Nanotubes: A Review on Growth Mechanism and Mass Production. Nanosci. and Nanoth. J., p. 3739-3758, 2010.

KUMAR, M. in Carbon Nanotubes - Synthesis, Characterization, Applications. Croácia: Editora InTech, 2011.

LI, Y.; ZHANG, X. B.; TAO, X.Y.; XU, J. M.; HUANG, W.Z.; LUO, J. H.; LUO, Z. Q.; LI, T.; LIU, F.; BAO, Y.; GEISE, H. J. Mass production of high-quality multi-walled carbon nanotube bundles on a Ni/Mo/MgO catalyst. Carbon, v. 43, p. 295-301, 2004.

LADEIRA, O. L., BR. Patent 000119, 2009.

MARCHESIN, M. S.; CECCI, R.; BRESSANIN, J. M.; BARTOLI, J. R.; ABE, I. Y.; CARREÑO, M. N. P.; PREYRA, I. IV Congresso Brasileiro de Energia Solar, 2012.

ODOM, T.W.; HUANG, J.L.; KIM, P.; LIEBER, C. M. Atomic structure and electronic properties of single-walled carbon nanotubes Nature, v. 391, p. 62 - 64, 1998.

RODRIGUES, J. G., Dissertação de mestrado, Universidade Federal de Minas Gerais, 2011.

Software Statística 7.0 - Empresa StatSoft.

ZHAN, S.; TIAN, Y.; CUI, Y.; WU, H.; WANG, Y.; YE, S.; CHEN, Y. Effect of process conditions on the synthesis of carbon nanotubes by catalytic decomposition of methane. China Particuology, v. 5, p. 213-219. 